\title{
Immune Mechanisms Underlying the Premunition Against Plasmodium falciparum Malaria
}

\author{
Jean Louis Pérignon, Pierre Druilhe
}

Laboratorie de Parasitologie Bio-Médicale, Institut Pasteur, 28 rue du Dr. Roux, and CHU-Necker, 75015 Paris, France

The most unique characteristic of a parasite when it is in its normal host is the ability to make itself tolerated, which clearly indicates that it has sophisticated means to ensure the neutrality of its host. This is true also in the case of Plasmodium falciparum, since after numerous malaria attacks an equilibrium is reached with a chronic stage of infection, characterized by a relatively low parasitemia, and low or no disease (Sergent \& Parrot 1935). We shall briefly review the main characteristics of this state of "premunition", and present data suggesting that the underlying mechanisms of defense rely on the cooperation between cell and antibodies, leading to an antibody dependent cellular inhibition of the intra-erythrocytic growth of the parasite.

Key words: malaria - Plasmodium falciparum - immunity - premunition

\section{IN VIVO OBSERVATIONS}

Premunition has the following characteristics:

-it is not a sterilizing type of immunity: chronic infection persists, although the maximal parasite load reached is low. Even if it adds only a little in terms of reduction of parasite load as compared to innate resistance, this additional immunity is substantial in terms of morbidity: it keeps the parasite load below the threshold of pathogenicity. Superinfection can occur, but it remains at low grade.

-it is seen in holo or hyperendemic areas, mainly in Africa (and in some places of Papua-New Guinea).

- the delay of acquisition is remarkably long, compared to the rate of transmission. Epidemiological studies in the above areas have helped to define three clinical periods: a short period of 0 to 5 years where mortality can occur; a longer period of 0 to 15-20 years where morbidity is frequent (though decreasing in frequency with age); thereafter a longer period of premunition where the disease in any form is usually absent. However, such epidemiological studies do not allow to distinguish the respective importance of the immunological competence of the subjects related to age, and of the long term exposure to the parasite, a point recently addressed by Baird et al. (1991).

-it seems independent of transmission levels provided it occurs at least once per year. -it is rapidly lost: exactly one year without rechallenge seems enough to loose this protective state.

-importantly, all available evidences suggest that premunition is strain independent.

-it is clearly IgG dependent, as shown by passive transfer experiments in humans (Cohen et al. 1961, Edozien et al. 1962, Sabchareon et al. 1991), which is the best available evidence that it relies on a true immune response to defined antigens.

-finally it appears to be the strongest type of immunity developed by humans against asexual blood stages (ABS) infection. It is therefore of utmost interest in order to gather an understanding of the underlying mechanisms and of their target antigens, which may help to define a strategy of vaccine development.

\section{IN VITRO DATA}

There are thus clear-cut evidences of the protective role of antibodies in vivo. This contrasts with the inability of in vitro assays to reliably detect a direct inhibitory effect of antibodies from protected subjects: we should remind that the pool of IgG from protected Africans that we used in our experiment of passive transfer in humans, had in vitro an enhancing effect upon cultured isolates upon which it was clinically effective in vivo (Bouharoun-Tayoun et al. 1990). This strongly supported the hypothesis 
that the effect of these antibodies could depend on their cooperation with effector cells. The set up of an in vitro assay relying on the cooperation of antibodies and cells, and able to detect protective antibodies, was undertaken in this laboratory more than ten years ago (Khusmith \& Druilhe 1983). It was first demonstrated by ourselves and others that merozoites and/or SIRBC were opsonized by macrophages, PMN and/or monocytes (Khusmith \& Druilhe 1982, Celada et al. 1983). Most interestingly, the presence of opsonizing antibodies correlated well with the state of protection (Druilhe \& Khusmith 1987). However, we found that opsonization by PMN did not influence the rate of parasite growth in vitro (Lunel \& Druilhe 1989). We further attempted to determine whether the cooperation of protective antibodies with various cell types may affect the survival of the parasite within the erythrocyte. We thus set up an assay of antibody-dependent cellular inhibition of parasite growth (ADCI) (Fig.). Various cell types, all from naive donors, were studied (Lunel \& Druilhe 1989): only monocytes were found effective while polymorphonuclear cells, as well as lymphocytes, platelets, and more surprisingly tissue macrophages were not. Most importantly, ADCI effectively discriminated protective from no protective antibodies, i.e. we found a good correlation at individual level (not only on the average) with the state of protective immunity.

More recent in vitro studies using the biological material derived from our IgG passive transfer experiment (Bouharoun-Tayoun et al. 1990) further supported the relevance of ADCI to protective immunity. As mentioned above, the purified IgG

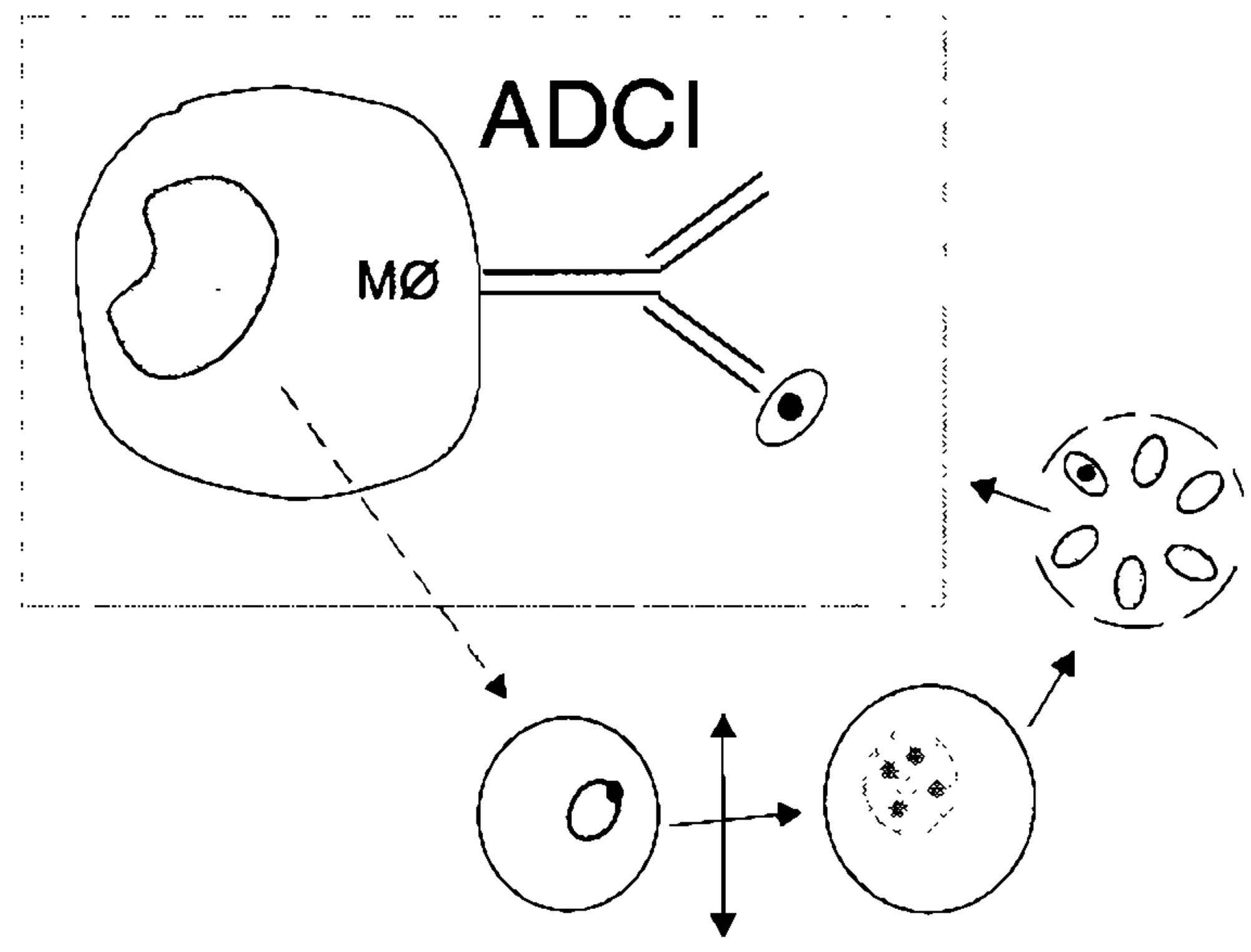

Schematic representation of the Antibody-Dependent Cellular Inhibition of parasite growth (ADCI). paradoxically enhanced the parasite growth; conversely no inhibition was due to the monocytes themselves; however a strong inhibition was observed when both were allowed to act together. This represented the first direct demonstration of an in vitro inhibitory effect of antibodies with proven in vivo protective efficacy.

Further unpublished material provide an indication of how ADCI functions: a) monocytes are triggered by cytophilic antibodies directed to a merozoite surface antigen and $b$ ) release soluble mediator(s) able to act at distance; c) this or these mediators, to date unidentified, block the division of surrounding intra-erythrocytic parasites at trophozoite stage; d) the effect is partially reversible.

These findings are in agreement with McGregor's observation that the delay between inoculation of Ig and the beginning of the decrease of parasitemia was not the same in all patients. He pin-pointed that the rupture of mature schizonts was required to trigger the mechanism mediated by antibodies in vivo, without being able to distinguish between schizonts and merozoites (McGregor 1964). Furthermore, a direct correlation was found in vivo between the parasite reduction rate induced by $\operatorname{IgG}$ and the initial level of parasitemia (Sabchareon et al. 1991) supporting the involvement of parasites in triggering ADCI.

- ADCI as a defense mechanism triggered by merozoites and acting upon intra-erythrocytic parasites, also provides an understanding of the chronicity of malaria. According to this model, the intra-erythrocytic parasite matures freely up to schizont stage, possibly for several cycles, until the number of released merozoites reaches the threshold sufficient to trigger some monocytes. The parasite being both the trigger and the target of ADCI, parasitemia will never go to 0 but will rather fluctuate at very low levels. This is indeed what is observed in vivo.

- Finally, ADCI is also consistent with an important feature of premunition mentioned above: the absence of strain specificity. Indeed, even mutants divoided of the specific targets to protective antibodies will be equally eliminated provided that the monocytes are triggered by "wild-type" parasites.

Thus ADCI is not only an in vitro assay that reliably detects protective antibodies, it may also be in vivo an efficient mechanism of defense against malaria parasites. 
Since ADCI relies only on those antibodies cytophilic to monocytes, we undertook to study in detail the distribution of Ig classes and IgG subclasses of antibodies directed against $P$. falciparum in sera from individuals with defined clinical states of resistance or susceptibility to malaria. This was performed on immunoblots so as to study the widest possible range of antibody specificities. Profound differences in the ratio of cytophilic versus noncytophilic antibodies were found: $\operatorname{IgG} 1$ and $\operatorname{IgG} 3$, two cytophilic isotypes, were found to predominate in protected subjects. Conversely, non cytophilic IgG2 and/or IgM predominated in non-protected subjects (Bouharoun-Tayoun \& Druilhe 1992). The function of total Ig presenting such an imbalance between cytophilic and non-cytophilic Ig was studied in vitro in ADCI assay. Not only did IgG from protected subjects cooperate efficiently with blood monocytes, whilst IgG from non-protected groups did not, but moreover the latter could inhibit the in vitro effect of the former suggesting that non-protected subjects had raised antibodies directed to epitopes critical for protection, but unable to trigger ADCI (Boucharoun-Tayoun \& Druilhe 1992). The demonstration that the protection against erythrocytic stages of $P$. falciparum is closely correlated with the production of cytophilic specific antibodies, provides the first clue to the understanding of the long delay of acquisition of protective immunity: this may not correspond to the progressive acquisition of responses to a wide pannel of antigens, as generally proposed, but rather to the switch from non-cytophilic to cytophilic antibody responses to a limited number of target antigens.

\section{CONCLUSION}

In humans, repeated infections by $P$. falciparum induce a progressive modulation of the immune response, eventually leading to an anti-parasite immunity characteristic of "premunition". This progressive modulation of the immune response, clearly exemplified by the acquisition of in vivo protective antibodies able to promote in vitro an ADCI effect, is important to consider, as it implies that antimalarial immunity should be assessed not only in quantitative terms (the higher titer, the best) but also from a qualitative point of view (the right antibody cooperating with the right cell).

\section{REFERENCES}

Baird JK, Jones TR, Danudirgo EW, Annis BA, Bangs MJ, Basri H, Purnomo, Masbar S 1991. Age-dependent acquired protection against Plasmodium falciparum in people having two years exposure to hyperendemic malaria. Am J Trop Med Hyg 45: 6576.

Bouharoun-Tayoun H, Attanath P, Sabchareon A, Chongsuphajaisiddhi T, Druilhe P 1990. Antibodies which protect man against $P$. falciparum blood stages do not on their own inhibit parasite growth and invasion in vitro but act in cooperation with monocytes. $J$ Exp Med 172: 1633-1641.

Bouharoun-Tayoun H, Druilhe P 1992. P. falciparum malaria: Evidence for an isotype imbalance which may be responsible for yhe delayed acquisition of protective immunity. Infect Immun 60: 1473-1481.

Celada A, Cruchard A, Perrin L 1983. Phagocytosis of $P$. falciparum parasitized erythrocytes by polymorphonuclear leukocytes. J Parasitol 69: 49.

Cohen S, McGregor IA, Carrington S 1961. Gamma globulin and acquired immunity to human malaria. $\mathrm{Na}$ ture 192:733-737.

Druilhe P, Khusmith S 1987. Epidemiological correlation between levels of antibodies promoting merozoite phagocytosis of $P$. falciparum and malaria immune status. Infect Immun 55: 888-891.

Edozien JC, Gilles HM, Udeozo IOK 1962. Adult and cord-blood gamma globulin and immunity to malaria in Nigerians. The Lancet II 1962: 951-955.

Khusmith S, Druilhe P 1982. Specific arming of human monocytes by cytophilic IgG enhances Plasmodium falciparum merozoite ingestion. 5th Intern. Cong. of Parasitol., Toronto, 1982. Mol Biochem Parasitol suppl 99.

Khusmith S, Druilhe P 1983. Cooperation between antibodies and monocytes that inhibit in-vitro proliferation of $P$. falciparum. Infect Immun 41: 219-224.

Lunel F, Druilhe P 1989. Effector cells involved in non specific and antibody-dependent mechanisms directed against $P$. falciparum blood stages in vitro. Infect Immun 57: 2043-2049.

McGregor IA 1964. The passive transfer of human malarial immunity. Am J Trop Med Hyg 13: 237-239.

Sabchareon A, Burnouf T, Ouattara D, Attanath P, Bouharoun H, Chantavanich P, Foucault C, Chongsuphajaisiddhi T, Druilhe P 1991. Parasitological and clinical human response to immunoglobulin administration in falciparum malaria. Am J Trop Med Hyg 45: 297-308.

Sergent ED, Parrot L 1935. L'Immunité, la Prémunition et la Résistance Innée. Archives de l'Institut Pasteur d'Algérie, TXIII: 279. 